\title{
Silicon photonic MEMS phase shifter with $\mu$ s time constant built on a foundry platform
}

\author{
Pierre Edinger ${ }^{1}$, Kristofer Kristinsson ${ }^{1}$, Carlos Errando-Herranz ${ }^{1}$, Alain Yuji \\ Takabayashi $^{2}$, Hamed Sattari ${ }^{2}$, Niels Quack ${ }^{2}$, Peter Verheyen ${ }^{3}$, Wim Bogaerts ${ }^{3,4}$, \\ and Kristinn B. Gylfason ${ }^{1}$ \\ ${ }^{1}$ KTH Royal Institute of Technology, Stockholm, Sweden; ${ }^{2}$ Ecole Polytechnique Fédérale de Lausanne, \\ Switzerland; ${ }^{3}$ Interuniversity Microelectronics Centre, Leuven, Belgium; ${ }^{4}$ Ghent University, Belgium \\ edinger@kth.se
}

\begin{abstract}
MEMS enable low power tuners in silicon photonics, but existing phase shifters lack in range, speed, and loss. We implement a $2 \pi$ phase shifter with a $1.54 \mu$ s time constant and $0.5 \mathrm{~dB}$ insertion loss in IMEC's iSiPP50G platform. (C) 2021 The Author(s)
\end{abstract}

\section{Introduction}

Silicon photonics is becoming increasingly relevant for new application domains of photonic integrated circuits (PICs), ranging from A.I. optimized hardware to quantum photonics [1,2]. Its potential for large-scale integration allows for complex circuits, but the scaling is often limited by variability, which is why circuits are being fitted with tunable elements. Also, programmable (PICs) are being pursued for creating more flexible photonics than achievable with current application specific circuits [3]. The tunable elements of PICs need to have a small footprint, short optical length, low optical loss, and low electrical power consumption. The mW level electrical power consumption of the thermo-optic phase shifters commonly employed [4] prohibits scaling to large circuits. Microelectromechanical systems (MEMS) offer $10^{4}$ lower power consumption than thermo-optic actuators, and crystalline silicon is an excellent mechanical material. This makes silicon MEMS, built into existing silicon photonics platforms, a promising technology to build the tunable elements of large scale PICs.

Here, we experimentally demonstrate a silicon photonic MEMS tunable phase shifter that achieves more than $2 \pi$ phase shift with a low insertion loss (IL) of $0.5 \mathrm{~dB}$ at a wavelength of $1550 \mathrm{~nm}$ using an actuation voltage of $25 \mathrm{~V}$. Moreover, due to its small size and low mass, the device has a large mechanical resonance frequency of $461 \mathrm{kHz}$ and operates without significant air damping. The $-3 \mathrm{~dB}$ bandwidth of the device is $648 \mathrm{kHz}$, corresponding to a time constant of $1.54 \mu \mathrm{s}$.

\section{Design and fabrication}

Our device relies on in-plane, electrostatic comb-drive actuators to physically adjust the mode cross-section in a suspended silicon waveguide. The devices are fabricated in IMEC's iSiPP50G silicon photonics process, followed by a MEMS release. Compared to our previously demonstrated design [5], we here show a device integrated in the full iSiPP50G platform, resulting in the final cross-section shown in Fig. 1 (a). The device layer is used both for the photonics and for the MEMS actuators. A low resistance path to the comb-drives, essential for high-speed operation, is provided by the two-level copper metalization and the selectively doped Si of iSiPP50G. The devices were implemented and tested using platform-standard bondpads and grating couplers, see Fig. 1 (b-c).
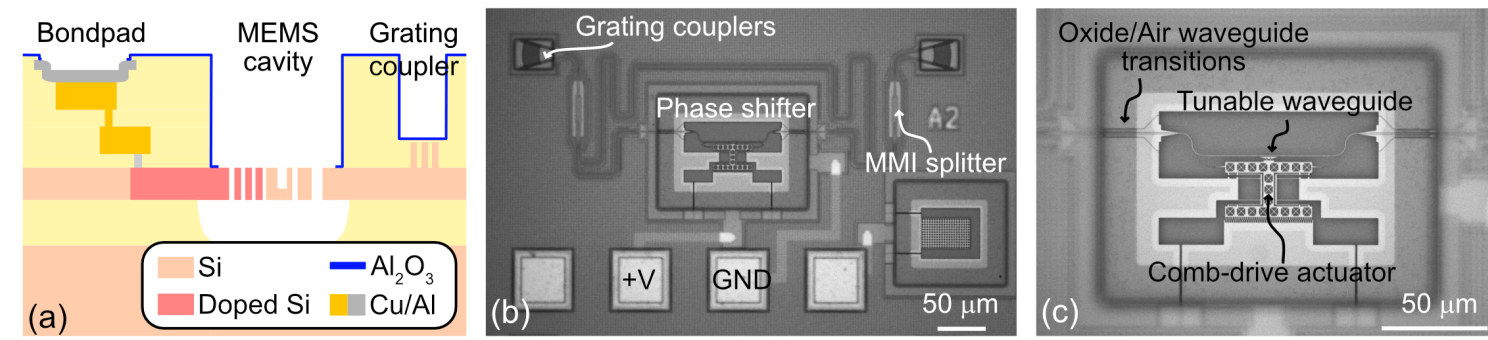

Fig. 1. Implementation. (a) The MEMS phase shifter is implemented in the device layer of IMEC's iSiPP50G. (b) Top view microscope capture of the phase shifter in a Mach-Zehnder interferometer. (c) Close up view of the MEMS phase shifter. 


\section{Measurements}

For testing, the phase shifter was included in one arm of an unbalanced integrated Mach-Zehnder interferometer (MZI). All interference fringes were fitted individually to obtain a map of phase shift and insertion loss over the wavelength range of our tunable laser as a function of voltage (Fig. 2 (a-b)). The insertion loss includes the MEMS phase shifter and two oxide- to air-clad waveguide transitions. To quantify the mechanical frequency response, a sinusoidal modulation $(8 \pm 0.5 \mathrm{~V}$ ) was applied to the electrostatic comb drive actuator and the resulting MZI output modulation and mechanical phase lag were measured using a lock-in amplifier, see Fig. 2 (c).
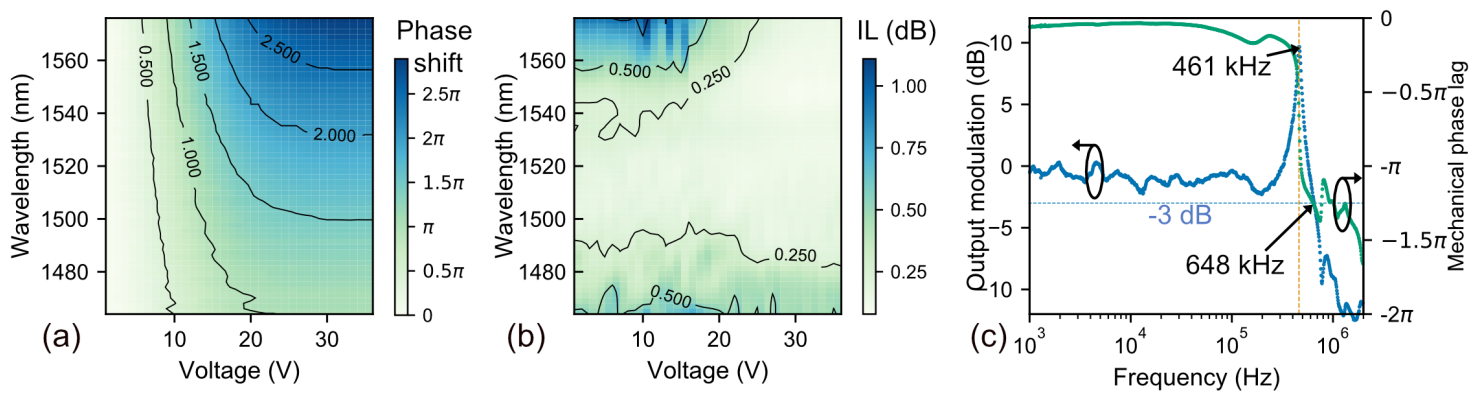

Fig. 2. Measurement results. (a-b) DC characterization of the device, with (a) phase shift with respect to applied bias and wavelength, and (b) insertion loss (IL) versus bias and wavelength. (c) AC characterization, with output amplitude and mechanical phase lag with respect to frequency.

\section{Conclusions}

We have demonstrated a photonic MEMS phase shifter implemented in a standard silicon photonics platform. The device achieves a full $2 \pi$ phase shift in a short optical length of 61 wavelengths (physical length of $50 \mu \mathrm{m}$ ), while only adding a loss of $0.5 \mathrm{~dB}$ to the circuit. This combination is essential for implementing programmable photonic circuits. Furthermore, the extremely low power consumption of below $1 \mu \mathrm{W}$ in static operation allows the implementation of reconfigurable on-chip optical meshes with more active devices than thermo-optics based programmable photonics. Finally, the rapid response time of these small MEMS devices makes them an exciting alternative for applications needing a large modulation amplitude and $10^{5} \mathrm{~Hz}$ range modulation speed, e.g. beamsteering for LIDAR, or active compensation of thermal fluctuations.

\section{Acknowledgements}

This work has received funding from the European Union's Horizon 2020 research and innovation programme under grant agreement No.780283 (MORPHIC). We thank Dr. Max Yan for access to measurement equipment.

\section{References}

1. Y. Shen, N. C. Harris, S. Skirlo, M. Prabhu, T. Baehr-Jones, M. Hochberg, X. Sun, S. Zhao, H. Larochelle, D. Englund, and M. Soljačić, "Deep learning with coherent nanophotonic circuits," Nature Photonics, vol. 11, pp. 441-446, July 2017. Number: 7 Publisher: Nature Publishing Group.

2. J. Wang, F. Sciarrino, A. Laing, and M. G. Thompson, "Integrated photonic quantum technologies," Nature Photonics, vol. 14, pp. 273-284, May 2020. Number: 5 Publisher: Nature Publishing Group.

3. W. Bogaerts, D. Perez, J. Capmany, D. A. B. Miller, J. Poon, D. Englund, F. Morichetti, and A. Melloni, "Programmable photonic circuits," Nature, vol. 586, pp. 207-216, Oct. 2020. Number: 7828 Publisher: Nature Publishing Group.

4. N. C. Harris, Y. Ma, J. Mower, T. Baehr-Jones, D. Englund, M. Hochberg, and C. Galland, "Efficient, compact and low loss thermo-optic phase shifter in silicon," Optics Express, vol. 22, pp. 10487-10493, May 2014. Publisher: Optical Society of America.

5. P. Edinger, C. Errando-Herranz, A. Y. Takabayashi, H. Sattari, N. Quack, P. Verheyen, W. Bogaerts, and K. B. Gylfason, "Compact low loss mems phase shifters for scalable field-programmable silicon photonics," in 2020 Conference on Lasers and Electro-Optics (CLEO), pp. 1-2, 2020. 